\title{
Common Spatial Pattern: An Improved Method for Atrial Fibrillation Wave Extraction
}

\author{
I Romero Legarreta, G Wübbeler, C Elster
}

Physikalisch-Technische Bundesanstalt, Berlin, Germany

\begin{abstract}
In this work, a spatial filtering by Common Spatial Pattern (CSP) is used for atrial fibrillation extraction. The CSP technique was applied by defining intervals in the ECG time series containing either the QRST signal or the remaining part of the ECG. This enabled a decomposition of the 12 lead ECG into 12 components sorted according to their similarity to the QRST signal.

A test database with simulated AF was generated. This test set was used to study the performance of several classical techniques and of the new method. The techniques considered were: CSP, Template subtraction, PCA and the ICA variants: SOBI, JADE, INFOMAX. For PCA, ICA and CSP, the subset of components that best matched the original AF signal was considered for each method. After testing the methods proposed, the one that performed best with our dataset was the CSP. The method that gave the worst results was the template subtraction.
\end{abstract}

\section{Introduction}

Atrial Fibrillation (AF) is probably the most frequent cardiac arrhythmia. The treatment of this pathology is complex and usually based on trial and error; treatment results are still unsatisfactory and unpredictable. For this reason the study of AF has received increasing interest in the scientific community during the last years.

The use of new digital processing techniques has permitted to extract the atrial activity for further analysis by proper cancellation of the ventricular activity [1]. For this purpose, different promising techniques have been proposed recently, e.g. spatiotemporal QRST cancellation (STC) by subtraction [2,3], application of Independent Component Analysis (ICA) [4,5] or Principal Component Analysis (PCA) [6,7]. A comparison study of these three main techniques: STC, ICA and PCA led to satisfactory results for this three methods [10]. However, the main difficulty in the application of ICA and PCA is the selection of the components that best match with the atrial source signal. Application of these cancellation methods permits the study of the atrial fibrillation wave in the frequency or time-frequency domain $[8,9]$.

After the extraction of the AF wave, the frequency analysis gives a better understanding of the phenomena involved in atrial fibrillation and its behaviour [9,11]. In addition, it can be utilized by cardiologists for diagnosis and therapy control $[12,13]$.

In this work we employ the technique of spatial filtering by Common Spatial Pattern (CSP) for the task of atrial fibrillation extraction. Spatial filters derived by CSP have been highly successfully applied for the classification of different brain signals in the framework of the brain-computer interface [14]. Here we adopt this technique in order to separate the ventricular activity from the remaining part of the ECG, possibly containing atrial fibrillation.

\section{Methods}

\section{A. Test AF signals}

For the developing and testing of an AF wave extraction method, a dataset of ECGs with simulated AF was created. An AF wave was simulated from a real AF ECG by manually canceling the segments corresponding to QRS-T waves. The gaps resulted were filled with the interpolation of adjacent AF segments. A QRS-T wave was obtained from real ECGs in sinus rhythm by manually canceling the $\mathrm{P}$ waves. The test signals were created by the addition of both AF and QRST waves. One example is shown in figure 1.

Thirty AF waves were simulated from selected real cases within the PTB AF database. Fourteen QRS-T waves were obtained from real cases within the PTB ECG database. The combination of them gave a total of 420 test signals. Each signal is comprised of the 12 standard leads with 10 seconds length. The sample frequency was $100 \mathrm{~Hz}$. 


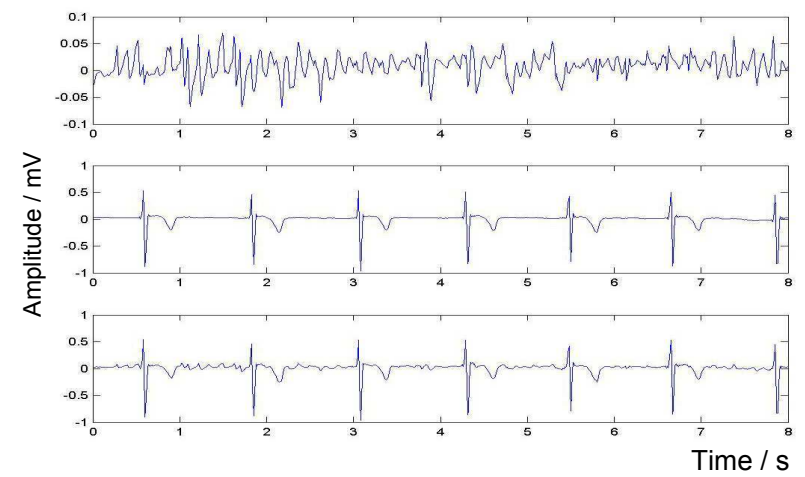

Figure 1. Generation of a test AF signal. The upper plot shows a simulated AF signal. The middle plot contains a QRS-T signal. The addition of both signals is showed in the lower plot.

\section{B. PTB AF database}

The PTB AF database comprises 546 cases classified as atrial fibrillation. After additional confirmation by experienced cardiologists four of these cases were removed yielding altogether 542 cases for the AFIB test database. The data were recorded with different sampling frequencies ranging from $400 \mathrm{~Hz}$ to $10 \mathrm{kHz}$. For the AFIB test database the data were downsampled (after low-pass filtering) resulting in a uniform sampling frequency of $100 \mathrm{~Hz}$. Additionally, the length of all
ECGs in the AFIB test database was restricted to 10 seconds duration.

\section{Common Spatial Pattern}

A usual PCA decomposition is based on the covariance matrix of the whole dataset and leads to components which are ranked by variance. The CSP technique expands this approach to the case were the multi-channel signal is assumed to contain the superposition of two different signals.

In order to apply the CSP technique to the ECG data two covariance matrices were estimated from the 12 lead ECG; one from the QRST-intervals ranging from $-100 \mathrm{~ms}$ to $300 \mathrm{~ms}$ around each R-peak, and the other from the remaining intermediate intervals. Using these covariance matrices the CSP algorithm determined directions in the 12 dimensional space that maximize the variance for the QRST signal and simultaneously minimize the variance of the remaining parts of the ECG. In this way the 12 lead ECG is decomposed into components which are ranked by their similarity to the QRST segment. By retaining only those components which display no significant QRST contribution a spatial filtering of the original 12 lead ECG is achieved which cancels most the ventricular activity. Figure 2 shows an example of a 12 lead ECG, decomposed and spatially filtered by CSP resulting in ECG traces with largely suppressed QRST.
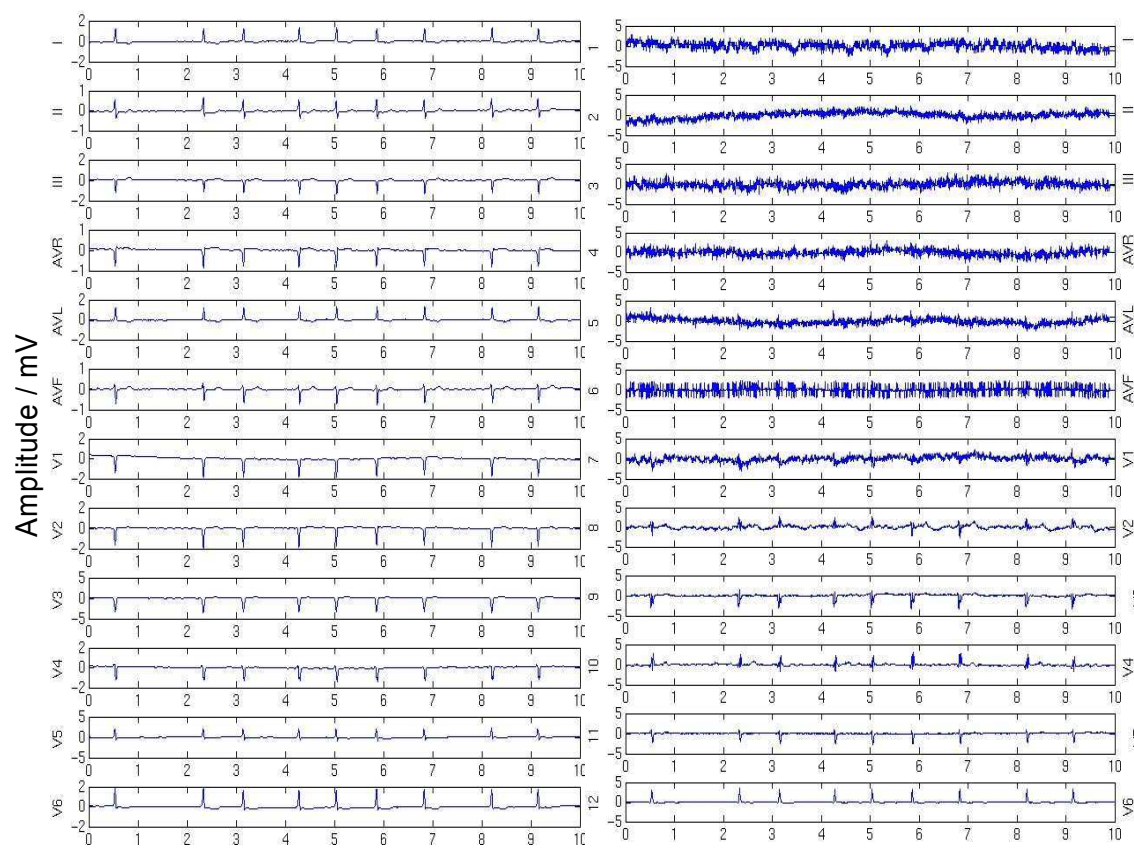

Time / s

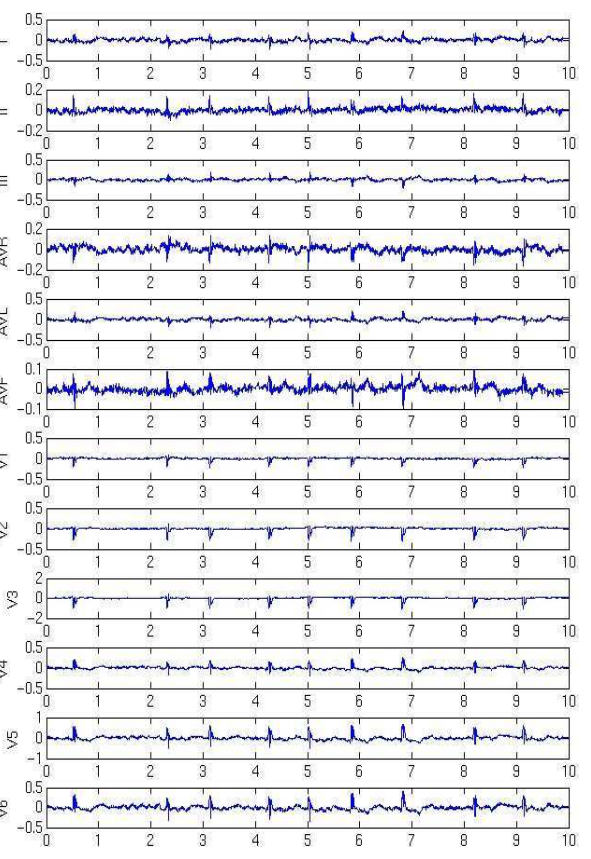

Figure 2. Application of the CSP-method to a standard 12 lead ECG. Left figure shows the original 12 lead ECG. The middle figure is based on the 12 components determined by CSP. The components 6 to 9 are used to calculate the spatially filtered 12 lead ECG (right figure). 


\section{Other methods.}

In addition to the method proposed for the authors in this paper, the CSP, other methods were also scrutinized and tested with the test signals generated. The methods investigated were the classical technique of the subtraction of a QRST template [15], PCA [1,6], and three variations of ICA: SOBI, JADE and INFOMAX [4].

\section{E. Component Selection}

The test dataset created was used to determine the performance of the different methods under analysis. For the methods PCA, CSP and the ICA variations the components were selected considering the optimal combination. After applying the methods to the signals, one single component was retained and the inverse method was applied in order to obtain the 12 standard leads. Both the estimated and the simulated AF waves were compared by means of the root mean squared error and the correlation coefficient. Only lead V1 was considered because it usually shows the best representation of the atrial activity [9]. In that way, all the 12 components were studied and then sorted with respect to the correlation value. Considering this new order the method was again applied by adding components in that order. Again, the inverse method was applied in order to obtain the 12 standard leads, and the estimated and the simulated AF waves were then compared by means of the root mean squared error and the correlation coefficient. The best correlation value was considered for each test signal.

\section{Results}

The 6 methods were applied to the total of 420 test ECGs generated. The output was compared to the original AF wave by means of their correlation. An example of the output of different methods can be seen in figure 3 .

The mean and median of the correlation values, together with the number of cases that obtained a correlation value higher than 0.5 obtained for each method are presented in Table 1 .

The method that gave the best performance with our dataset was the CSP with an average (median) correlation value of $0.41(0.38)$; for $27 \%$ of the simulated ECGs a correlation value higher than 0.5 was observed. JADE and PCA performed similarly (mean (median) of 0.37 (0.34) for JADE and 0.36 (0.34) for PCA). SOBI gave an average (median) correlation value of 0.35 (0.30), INFOMAX $0.32(0.30)$ and finally the worst results were obtained after applying the technique of template subtraction with a mean (median) of 0.29 (0.27).
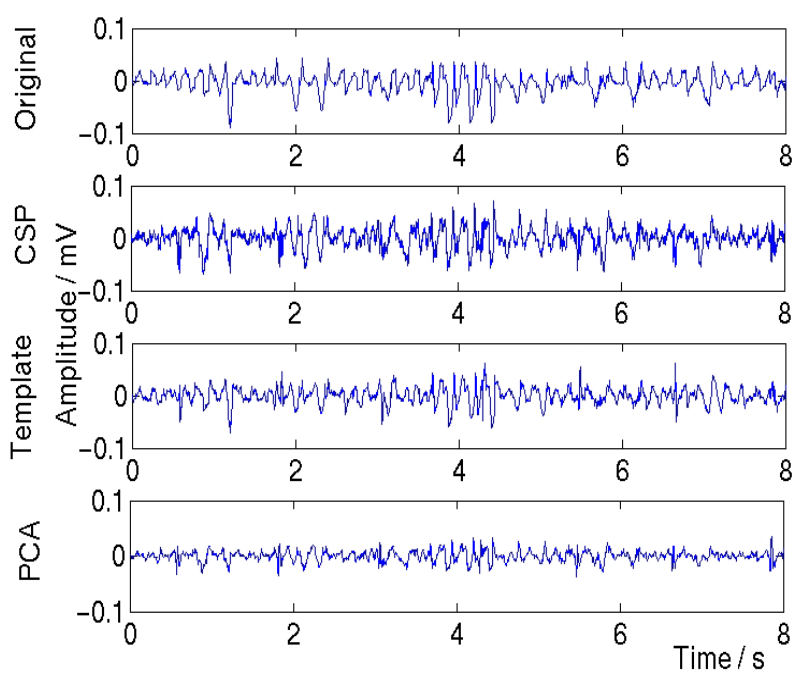

Figure 3. Comparison of different AF waves. The first plot shows the original AF used to generate a test signal, the other plots are the output signal corresponding to the CSP, template and PCA methods.

Table 1. Mean and Median of the correlation values together with the percentage of values higher than 0.5 .

\begin{tabular}{|l|c|c|c|}
\hline Method & Mean & Median & Cases $>\mathbf{0 . 5}(\mathbf{\%})$ \\
\hline Template & 0.30 & 0.28 & $8 \%$ \\
\hline PCA & 0.36 & 0.34 & $18 \%$ \\
\hline CSP & 0.41 & 0.38 & $27 \%$ \\
\hline SOBI & 0.35 & 0.31 & $21 \%$ \\
\hline JADE & 0.37 & 0.34 & $19 \%$ \\
\hline INFOMAX & 0.32 & 0.30 & $16 \%$ \\
\hline
\end{tabular}

The difference between the correlation values obtained by CSP and JADE (second best method) was found highly significant $(\mathrm{p}<3 \times 10-19)$.

\section{Discussion and conclusions}

A new method for the extraction of the AF wave is presented in this paper: the Common Spatial Filter method. This method consist in the decomposition of the 12 standard lead ECG in 12 components sorted according to their similarity to the QRST signal. This permits to filter out those components that are more similar to the QRS-T waves, retaining those that have the information of the atrial activity. This method was tested and compared with other classical method like PCA, ICA and template subtraction.

As a conclusion, the new method (CSP) for AF extraction performed on our test database better than the considered techniques. 


\section{Acknowledgements}

The authors thanks Prof. Andreas Bollmann and Dr. Daniela Husser for the careful re-validation of the AFIB test data, and also Dr. Dieter Kreiseler and Dr. RalfDieter Bousseljot for providing the ECG data for this work.

This work was supported by the Deutscher Akademischer Austausch Dienst (DAAD).

\section{References}

[1] Romero Legarreta I, Component Selection for PCA-based Extraction of Atrial Fibrillation. Computers in Cardiology 2006; 33: 137-140.

[2] Stridh M, Sörnmo L. Spatiotemporal QRST Cancellation Techniques for Analysis of Atrial Fibrillation: Methods and Performance. Computers in Cardiology 1998; 25:633636.

[3] Stridh M, Sörnmo L. Spatiotemporal QRST Cancellation Techniques for Analysis of Atrial Fibrillation. IEEE Transactions on Biomedical Engineering 2001; 48(1):150111.

[4] Rieta JJ, Zarzoso V, Millet Roig J, García Civera R, Ruiz Granell R. Atrial Activity Extraction Based on Blind Source Separation as an Alternative to QRST Cancellation for Atrial Fibrillation Analysis. Computers in Cardiology 2000; 27:69-72.

[5] Steinhoff U. Signal Identification and Noise Suppression in Multi-Channel ECG and MCG by Independent Component Analysis (ICA). In: De Ambroggi L, Katila T, Maniewski R. High Resolution ECG and MCG mapping 2003: Warsaw: International Centre of Biocybernetics: 117-125.

[6] Langley P, Bourke JP, Murray A. Frequency Analysis of Atrial Fibrillation. Computers in Cardiology 2000; 27: 6568.

[7] Raine D, Langley P, Murray A, Furniss SS, Bourke JP. Surface Atrial Frequency Analysis in Patients with Atrial Fibrillation. Journal of Cardiovascular Electrophysiology 2005; 16(8): 838-844.

[8] Langley P, Stridh M, Rieta JJ, Sörnmo L, Millet-Roig J, Murray A. Comparison of Atrial Rhythm Extraction Techniques for the Estimation of the Main Atrial Frequency from the 12-lead Electrocardiogram in Atrial Fibrillation. Computers in Cardiology 2002;29:29-32.

[9] Stridh M, Sörnmo L, Meurling C, Olsson B. Frequency trends of atrial fibrillation using the surface ECG. Proc. EMBS, IEEE Engineering in Medicine and Biology Society, Atlanta, USA, 1999..

[10] Langley P, Rieta JJ, Stridh M, Millet-Roig J, Sörnmo L, Murray A. Comparison of Atrial Signal Extraction Algorithm in 12-Lead ECGs with Atrial Fibrillation. IEEE Transactions on Biomedical Engineering 2006; 53(2): 343346.

[11] Langley P, Steinhoff U, Trahms L, Oeff M, Murray A. Analysis of Spatial Variation in the Atrial Fibrillation Frequency from the Multi-channel Magnetocardiogram. Computers in Cardiology 2003; 30: 137-140.

[12] Husser D, Stridh M, Sörnmo L, Olsson B, Bollmann A.
Frequency Analysis of Atrial Fibrillation From the Surface Electrocardiogram. Indian Pacing and Electrophysiology Journal 2004; 4(3): 122-136.

[13] Bollmann A, Kanuru NK, McTeague KK, Walter PF, DeLurgio DB, Langberg JJ. Frequency Analysis of Human Atrial Fibrillation Using the Surface Electrocardiogram and Its Response to Ibutilide. American Journal of Cardiology 1998; 81: 1439-1445.

[14] Müller-Gerking J, Pfurtscheller $G$ and Flyvbjerg $H$. Designing optimal spatial filters for single-trial EEG classification in a movement task. Clin. Neurophysiol. 1999; 110: 787-798

[15] Shah DC, Haissaguerre M, Yamane T, et al. Unmasking the ECG morphology of short coupled atrial ectopics by adjacent QRST subtraction. Pacing Clin Electrophysiol. 2001;24:651Type your references here. With the reference style, numbering is supplied automatically.

Address for correspondence

Iñaki Romero Legarreta

PTB - AG 8.41

Abbestr. 2-12

10587 Berlin (Germany)

inaki.romero@ptb.de 